\section{IN THE NEWS}

\section{Monkey talk}

Claims that a pygmy chimp called Kanzi has developed the ability to talk hit the headlines around the world when they were published in the New Scientist (UK, 2 January 2003). Although the $B B C$ Online maintained an air of quiet scepticism "Ape 'learns to talk"' - others were more enthusiastic, with the Times of India (2 January 2003) proclaiming "Speaking chimpanzee leaves experts amazed".

The researchers working with Kanzi, Jared Taglialatela and Sue Savage-Rumbaugh of Georgia State University, claim that the chimp spontaneously started making four distinct sounds, corresponding to the words 'banana', 'grapes', 'juice' and 'yes'. Kanzi, like other primates, can communicate by pointing at symbols - but this is the first report of an ape making sounds that have distinct meanings across different situations. According to the Straits Times (Singapore, 3 January 2003), the claims "...challenge the long-held belief that apes have no language ability."

As the New Zealand Herald (4 January 2003) points out, scientists disagree over what constitutes 'language'.

"Some linguists believe that even symbolic

communication, which many chimps achieve, qualifies as language, but many now say some mastery of syntax is also required." In the New Scientist, Frans de Waal of Emory University spoke for the primatology community when he said, "Sometimes we feel it's a bit unfair that [linguists] move the goalposts as soon as we get near."

If the claims hold up, Kanzi will become as famous as Washoe, the chimp who first learned American Sign Language. Primatologist John Mitani of the University of Michigan commented, "There have to be evolutionary precursors to what we do. We are beginning to find them in the primate world." (New Zealand Herald).

\section{Eyes wide shut}

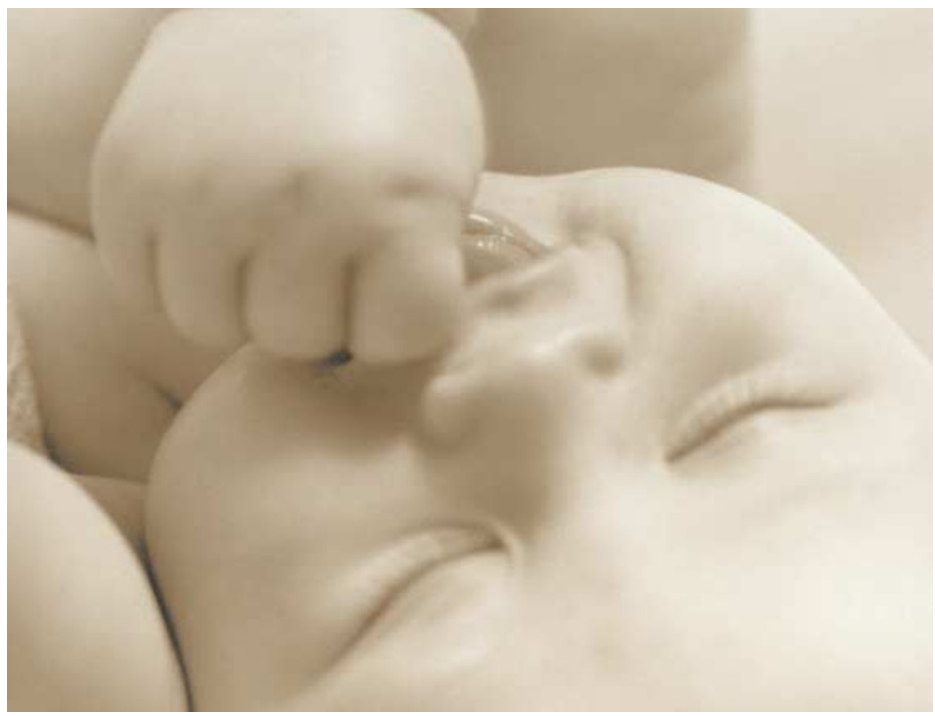

The unopened eyes of some newborn mammals might be more sensitive to light than was previously thought, according to a report by Akerman et al. in Neuron.

It was already known that very bright or high-contrast stimuli can elicit a response in the visual system before eye opening - for example, the eyes of a ferret can respond to light as early as postnatal day (P) 19 , even though they do not open until around P32. However, the effects of natural levels of light on the unopened eye have not been explored until now.
Akerman et al. presented young ferrets (P20-P26) with movies that simulated the types of visual stimuli that they would be exposed to in their normal rearing environment. They measured the activity of neurons in the lateral geniculate nucleus (LGN), which relays visual information from the retina to the visual cortex. Surprisingly, instead of seeing random spontaneous activity, the authors found that the LGN neurons showed patterns of activity that were consistent with a specific response to the light stimulus. This indicated that the retinal ganglion cells (RGCs) that project to this nucleus were transmitting meaningful information.

This study also shed new light on activity-dependent processes during visual system development. The authors examined the development of projections to the LGN from two types of RGC - 'On' cells, which respond to increases in light intensity, and 'Off' cells, which respond to decreases in light intensity. Usually, during early postnatal development, the On and Off LGN afferents segregate, so that individual LGN neurons come to receive inputs from only one cell type. However, in

\title{
NEUROLOGICAL DISORDERS
}

\section{Protein therapeutics}

Using large molecules such as proteins for the treatment of neurological disorders is a relatively unexplored idea, owing in part to the problems associated with their delivery to the nervous system. Two recent papers significantly encourage the development of protein-based therapies by highlighting their potential in rodent models of ischaemia and Alzheimer's disease.

In the first paper, Asoh et al. report the construction of a powerful antiapoptotic protein that can readily enter cells. They engineered the molecule by fusing the protein transduction domain (PTD) of the HIV/Tat protein with a mutant form of Bcl- $x_{\perp}$ (FNK) that has a more powerful anti-apoptotic effect than the wild-type protein. The authors obtained evidence that this hybrid molecule could enter cultured neurons and protect them from apoptotic stimuli. More importantly, a prospective intraperitoneal injection of PTD-FNK to gerbils prevented neuronal death in the hippocampus after an episode of ischaemia. These results confirm and extend observations published by Cao et al. using wild-type $\mathrm{Bcl}-\mathrm{x}_{\mathrm{L}}$. It will now be important to test whether a similar beneficial effect can be obtained if
PTD-FNK is administered after the ischaemic insult.

In the second paper, Matsuoka et al. explored the possibility that a peripherally administered protein that binds to the $\beta$-amyloid peptide $(\mathrm{A} \beta)$ can decrease the brain amyloid burden. Inspired by previous observations by DeMattos et al., which showed that the peripheral administration of an $A \beta$ antibody reduces brain $\mathrm{A} \beta$, Matsuoka et al. injected the $A \beta$-binding protein gelsolin to $\mathrm{A} \beta$-producing mutant mice. They found that this treatment reduced the brain level of $A \beta$ and its accumulation in plaques in young mice, and that the ganglioside GM1 (another large molecule that binds to $A \beta$ ) had a similar effect. As the 
ferrets that were reared in the dark from P16, some LGN neurons were found to respond to both increases and decreases in luminance. This implies that light is required for the normal segregation of the On and Off afferents.

The role of visual experience in the early sculpting of the visual system is still controversial, and this report is likely to add further fuel to the debate. There is little question that neuronal activity has a role, but the events that occur before eye opening were assumed to be driven by spontaneous activity, rather than stimulation of the nervous system by light. These new observations raise the possibility that some of the effects that were formerly attributed to spontaneous activity might actually result from visual experience.

Heather Wood

(2) References and links ORIGINAL RESEARCH PAPER Akerman, $\mathrm{C}$. J. et al. Visual experience before eye-opening and the development of the retinogeniculate pathway. Neuron 36, 869-879 (2002)

FURTHER READING Wong R. O. L \& Ghosh, A Activity-dependent regulation of dendritic growth and patterning. Nature Rev. Neurosci. 3, 803-812 (2002)

\section{WEB SITES}

Encyclopedia of Life Sciences:

http://www.els.net/

neural activity and the development of brain circuits

blood-brain barrier is essentially impermeable to both gelsolin and GM1, these molecules probably act as peripheral sinks of $A \beta$.

The two papers converge on the idea that protein therapeutics might be a promising strategy to treat neurological disorders. Whether such a promise will be fulfilled in the clinical arena is still open to question.

Juan Carlos López

(1) References and links ORIGINAL RESEARCH PAPERS Asoh, S. et al. Protection against ischemic brain injury by protein therapeutics. Proc. Natl Acad. Sci. USA 99, 17107-17112 (2002) | Matsuoka, Y. et al. Novel therapeutic approach for the treatment of Alzheimer's disease by peripheral administration of agents with an affinity to $\beta$-amyloid. J. Neurosci. 23, 29-33 (2003) FURTHER READING Cao, G. et al. In vivo delivery of a Bcl- $x$ f fusion protein containing the TAT protein transduction domain protects against ischemic brain injury and neuronal apoptosis. J. Neurosci. 22, 5423-5431 (2002) | DeMattos, R. B. et al. Peripheral anti-A $\beta$ antibody alters CNS and plasma $A \beta$ clearance and decreases brain $A \beta$ burden in a mouse model of Alzheimer's disease. Proc. Natl Acad. Sci. USA 98, 8850-8855 (2001)

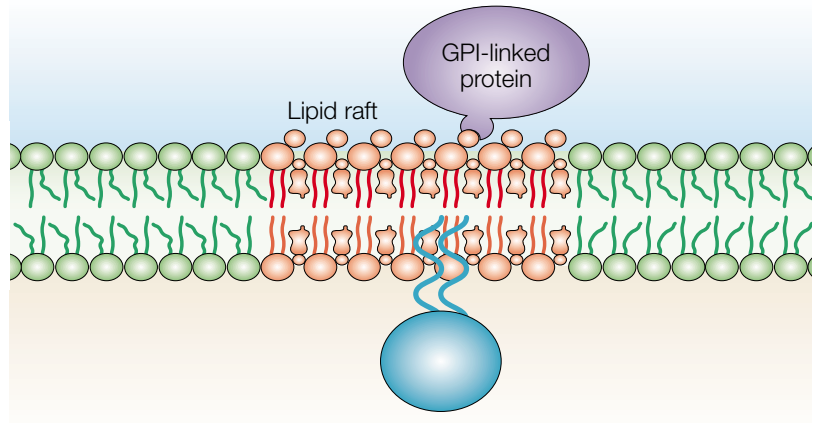

CELL BIOLOGY OF THE NEURON

\section{Growth cones go rafting}

New insights into the regulation of growth cone motility by cell adhesion molecules (CAMs) come from a study published in the Journal of Cell Biology. Nakai and Kamiguchi show that certain CAMs are localized to specialized microdomains within the cell membrane, known as lipid rafts, and that these rafts are important for growth cone migration.

Lipid rafts are regions of membrane into which cholesterol and sphingolipids are preferentially packaged. Specific proteins can attach to these areas, allowing regional concentration of signalling molecules. By separating out the lipid rafts, or detergent-resistant membranes (DRMs), from mouse cerebellar neurons, the authors found that two specific CAMS - L1 and $\mathrm{N}$-cadherin ( $\mathrm{N}$-cad) - were expressed in DRMs (as well as elsewhere in the membrane). Another CAM, $\beta 1$ integrin, was found only in non-DRM fractions of cell membrane.

Is this localization of $\mathrm{L} 1$ and $\mathrm{N}$-cad to DRMs essential for their function in mediating growth cone motility? Nakai and Kamiguchi used various methods of disrupting DRMs in living neurons to show that such disruption prevented growth cones from moving on a substrate of $\mathrm{L} 1$ or N-cad (both CAMs show homophilic binding, so that growth on an L1 substrate, for example, is mediated by L1 in the growth cone). Growth cone motility on a laminin substrate, to which $\beta 1$ integrin binds, was unaffected by DRM disruption.

To look at regional requirements for DRMs within the growth cone, the authors used a technique known as micro-scale chromophore-assisted laser inactivation (micro-CALI). A target molecule, in this case GM1 gangliosides (a marker for lipid rafts), is labelled with a dye-conjugated ligand. When a part of the cell is then irradiated with a laser, the labelled molecules and their immediate neighbours are selectively perturbed. Disruption of DRMs in growth cones by micro-CALI impaired growth cone motility mediated by $\mathrm{L} 1$ or $\mathrm{N}$-cad, but not by $\beta 1$ integrin. By irradiating just part of the growth cone, Nakai and Kamiguchi showed that DRMs were needed in the periphery of the growth cone, but not the central domain, for growth cone motility.

These results fit well with previous studies showing that these two domains within the growth cone probably have distinct functions in growth cone guidance, with the peripheral domain generating instructive signals and the central domain being responsible for more permissive signals. This elegant study constitutes one of the best proofs of a functional role for lipid rafts in neurons.

Rachel Jones

(0) References and links

ORIGINAL RESEARCH PAPER Nakai, Y. \& Kamiguchi, H. Migration of nerve growth cones requires detergent-resistant membranes in a spatially defined and substrate-dependent manner. J. Cell Biol. 159, 1097-1108 (2002)

FURTHER READING Simons, K. \& Toomre, D. Lipid rafts and signal transduction. Nature Rev. Mol. Cell Biol. 1, 31-39 (2000)
H I G H L I G H T S

LEARNING AND MEMORY

\section{Family ties}

Sheep are a good model to study social recognition memory - they form a strong family bond with their offspring shortly after parturition and strongly reject other newborn lambs. Olfactory cues are crucial for this form of memory, particularly during the early stages, but auditory and visual stimuli are also relevant later on. How is the different sensory information processed and integrated? A recent paper begins to address this issue by looking for transcriptional changes that might be associated with recognition memory in sheep.

The authors focused their efforts on brain-derived neurotrophic factor (BDNF), as this molecule seems to participate in plastic processes that might underlie memory formation. They looked for BDNF mRNA 4.5 hours post partum. At this time, an olfactory memory is already expressed, but the consolidation of the recognition memory is still ongoing. The authors saw that, in addition to structures of the olfactory processing system, BDNF mRNA was found in regions of the temporal, entorhinal, anterior cingulate and frontal cortices, as well as in the hippocampus and the diagonal band of Broca. As these structures have been implicated in attentional processes and visual recognition in some species, the authors speculate that the increase in BDNF mRNA levels reflects the reorganization of neural circuits in these regions at the time of memory formation. Although it is early to draw any definitive conclusions, these results point to several brain regions that we now need to probe in search of the mechanisms that underlie social recognition memory.

Juan Carlos López

6) References and links ORIGINAL RESEARCH PAPER Broad, K. D. et al. Increased BDNF and trk-B mRNA expression in cortical and limbic regins following formation of a social recognition memory. Eur. J. Neurosci. 16, 2166-2174 (2002)

FURTHER READING Insel, T. R. \& Young L. J. The neurobiology of attachment. Nature Rev. Neurosci. 2, 129-136 (2001) 